\title{
Correction to: Pointwise agglomeration in continuous racetrack model
}

\section{Kensuke Ohtake ${ }^{1} \cdot$ Atsushi Yagi $^{2}$}

Published online: 19 May 2021

C ISEG - Instituto Superior de Economia e Gestão 2021

\section{Correction to: Portuguese Economic Journal https://doi.org/10.1007/s10258-021-00197-5}

Equation 1 is incorrect in the original article, the correct Eq. 1 should be the below:

$$
\left\{\begin{aligned}
w(t, x)= & {\left[\mu \int_{S} w(t, y) G(t, y)^{\sigma-1} e^{-(\sigma-1) \tau|x-y|} d \Lambda(t, y)\right.} \\
& \left.+\frac{1-\mu}{2 \pi} \int_{S} G(t, y)^{\sigma-1} e^{-(\sigma-1) \tau|x-y|} d y\right]^{\frac{1}{\sigma}} \\
G(t, x)= & {\left[\int_{S} w(t, y)^{1-\sigma} e^{-(\sigma-1) \tau|x-y|} d \Lambda(t, y)\right]^{\frac{1}{1-\sigma}} } \\
\omega(t, x)= & w(t, x) G(t, x)^{-\mu} \\
\frac{\partial \Lambda}{\partial t}(t, x)= & \gamma\left[\omega(t, x)-\int_{S} \omega(t, y) d \Lambda(t, y)\right] \Lambda(t, x)
\end{aligned}\right.
$$

The original article has been corrected.

Publisher's Note Springer Nature remains neutral with regard to jurisdictional claims in published maps and institutional affiliations.

The original article can be found online at https://doi.org/10.1007/s10258-021-00197-5.

Kensuke Ohtake

k-ohtake@sigmath.es.osaka-u.ac.jp

1 Center for Mathematical Modeling and Data Science, Osaka University, Toyonaka, Osaka 560-8531, Japan

2 Graduate School of Information Science and Technology, Osaka University, Professor Emeritus, Suita, Osaka 565-0871, Japan 